\title{
The impact of scoring system in the evaluation of Nonalcoholic fatty liver disease (NAFLD) and nonalcoholic steatohepatitis (NASH)
}

\begin{abstract}
Nonalcoholic fatty liver disease (NAFLD) is an increasingly prevalent disease that has become the leading cause of liver-related morbidity and mortality in industrialized countries. It encompasses a spectrum of pathological manifestations that range from fatty infiltration without liver damage, to inflammation which can progress to fibrosis and cirrhosis. Individuals with features of the metabolic syndrome are at high risk of developing NAFLD. A major challenge is to find the reliable non-invasive diagnostic tool for the different aspects of NAFLD, particularly steatosis, steatohepatitis, and fibrosis. Currently, a liver biopsy is the definitive diagnostic test, but it is invasive and carries the risk of overt complications, and provides information on a very small portion of the liver. In this review, we discussed the various scores for diagnostic evaluation of patients with NAFLD and NASH.
\end{abstract}

Keywords: nonalcoholic fatty liver disease, nonalcoholic steatohepatitis, fibrosis, diagnostic score
Volume 10 Issue 5 - 2019

\author{
Muhammad Nadeem Yousaf, ${ }^{1,2}$ Fizah S \\ Chaudhary, ${ }^{3}$ Wajahat Z Mehal ${ }^{2,4}$ \\ 'Department of Internal Medicine, Union Memorial Hospital, \\ USA \\ ${ }^{2}$ Department of Internal Medicine, Section of Digestive Diseases, \\ Yale School of Medicine, USA \\ ${ }^{3}$ American University of Barbados, USA \\ ${ }^{4}$ Digestive Diseases, VA Hospital, USA
}

\begin{abstract}
Correspondence: Muhammad Nadeem Yousaf, Department of Internal Medicine, MedStar Union Memorial Hospital, Baltimore, 9000 Franklin Square drive, Primary care center, Second floor, Baltimore, MD 21237, Tel 443-777-8300, Fax 443-777-7869, Email Muhammad.n.yousaf@medstar.net
\end{abstract}

Received:September 16,2019 | Published: October 16, 2019

\begin{abstract}
Abbreviations: NAFLD, nonalcoholic fatty liver disease; NAFL, non-alcoholic fatty liver; NASH, non-alcoholic steatohepatitis; NFS, fibrosis score; AURCO, area under the receiver operating characteristic curve; CI, comprehensive index; SNPs, single nucleotide polymorphisms; FLI, fatty liver index; ELFP, enhanced liver fibrosis panel
\end{abstract}

\section{Introduction}

Nonalcoholic fatty liver disease (NAFLD) is one of the most common etiologies of liver diseases in the United States. ${ }^{1}$ NAFLD is a spectrum of pathological manifestations in non-alcoholic individuals which range from fatty infiltration of liver to steatohepatitis and cirrhosis. It is further categorized into non-alcoholic fatty liver (NAFL) and non-alcoholic steatohepatitis (NASH) based on histological findings. NAFL is defined as the presence of $\geq 5 \%$ hepatic steatosis without evidence of hepatocytic injury while NASH is defined as the presence of $\geq 5 \%$ hepatic steatosis and inflammation with hepatocyte ballooning injury with or without fibrosis., ${ }^{1,2}$ NAFLD is a highly prevalent disease and has been reported to affect about one billion individuals in the world. ${ }^{3}$ Recent studies suggest that up to 30 to $40 \%$ of Americans have NAFLD in the United States. ${ }^{4-9}$ Approximately $20 \%$ of NAFLD affected individuals are at risk of progression to NASH which is the second most common etiology for liver transplantation in the United States and is expected to be the leading cause in the next few years. ${ }^{10,11}$ Screening for NAFLD is challenging because most patients are asymptomatic until the development of cirrhosis. Asymptomatic individuals come to attention due to blood tests performed for other indications. ${ }^{12}$ Compared to the general population, patients with NAFLD have a significantly higher all-cause mortality, an increased incidence of cancer, diabetes and cardiovascular diseases, which is also the most common cause of death in pre-cirrhotic NAFLD. ${ }^{1,13,18}$ Considering these challenges, there is a need to establish a practical and effective approach for the evaluation and early detection of NAFLD particularly in those individuals who are at risk of developing fibrosis. Various biochemical marker and imaging modalities are being used for the diagnostic evaluation of both NAFLD and NASH. In this review we focused on the role of various scoring system in the evaluation of NAFLD and its progression to NASH and advanced liver fibrosis.

\section{The NAFLD fibrosis score (NFS)}

NFS is commonly used scoring system to estimate advanced liver fibrosis. The NFS can only be used to determine the severity of liver fibrosis rather than diagnosis of NASH. ${ }^{2}$ The NFS is based on the following parameters; age, BMI, hyperglycemia, albumin, platelet count and AST/ALT ratio. Using these 6 parameters, Angulo et al. differentiate between advanced and minimal fibrosis with an area under the receiver operating characteristic curve (AURCO) 0.88 and 0.82 in the estimation and validation group respectively. ${ }^{19}$ They also determined a cut off value of NFS less than -1.455 to exclude and greater than 0.676 to predict advanced liver fibrosis. Using low cut off value, the fibrosis was excluded with high accuracy (NPV up to 93\%), while fibrosis was diagnosed using high cutoff value with high accuracy (PPV up to $90 \%$ ). Although the precision rate is very high to predict or exclude advanced fibrosis $(\geq \mathrm{F} 3)$ using these cutoff values, however NFS does not clear the stage of liver fibrosis (F1-2) if the value is between -1.455 to 0.676 . Liver biopsy is needed in these cases of intermediate stage of liver fibrosis. Further studies are needed to overcome these limitations of NFS in the differentiation of steatosis and NASH. 


\section{Fibrosis-4 (FIB-4) index}

FIB-4 index is a non-invasive method to determine advances fibrosis in NAFLD and is calculated by documenting the age of a patient and the values of AST, ALT and platelet count obtained from a routine blood test, which emphasizes the ease of obtaining the FIB-4 score in a patient. A recent retrospective cohort study suggests that the FIB-4 index can provide a definitive diagnosis of NASH with a $67 \%$ sensitivity and $73 \%$ specificity. The same study also concluded that FIB-4 is also well equipped to identify NASH with mild fibrosis out of a NAFLD study population ( $57 \%$ sensitivity, and $75 \%$ specificity). ${ }^{20} \mathrm{~A}$ recent study determined the cutoff value of FIB-4 score for evaluation of advanced fibrosis. ${ }^{21} \mathrm{~A}$ cutoff value of $<1.45$ excludes advanced fibrosis and has 74\% sensitivity, 71\% specificity, 22\% PPV, 73\% NPV giving an AUROC of 0.87. Similarly, a higher cutoff value $>3.25$ predicts advanced fibrosis and has 26\% sensitivity, 98\% specificity, $75 \% \mathrm{PPV}, 85 \% \mathrm{NPV}$ giving an AUROC of 0.88 . The efficiency of FIB-4 score between 1.45 to 3.25 in still undetermined and there is a scarcity of studies that have assessed the efficacy of FIB-4 in the clinical setting. Further studies need to be performed to determine its usefulness in diagnosing NASH and NAFLD. For detection of advanced liver fibrosis (F3) or cirrhosis (F4), both NFS and FIB-4 index are recommended to be useful tools in current guidelines. ${ }^{1}$ Both NFS and FIB-4 are equivalent to MRE and better than other indices (like ASL/ALT ratio, BRAD score) for detection of advanced fibrosis in biopsy-proven NAFLD patients. ${ }^{22}$

\section{Comprehensive index $(\mathrm{Cl})$}

The comprehensive index (CI) combines six different serum biomarkers (weight, BMI, waist circumference, AST/ALT, triglycerides and fasting blood glucose) with different anthropometric denominations via a multivariate logistic regression analysis to detect NAFLD at an earlier stage. The sensitivity of CI was $90 \%$ while the specificity was $76 \%{ }^{23}$ The CI can also take into account the development of single nucleotide polymorphisms (SNPs) in genes that regulate lipid metabolism. The incorporation of known gene mutations in CI can further enhance its sensitivity and specificity for detection of NAFLD. CI is unable to discern between various pathologic stages of NAFLD such as simple fatty liver, hepatic steatosis and its complicated advancements such as liver cirrhosis and $\mathrm{HCC}$, which explains the narrow use of this index in current practice.

\section{Fatty liver index (FLI)}

FLI is a simple and one of an accurate predictor of hepatic steatosis in the general population. It is based on an algorithm that accounts for four parameters including BMI, waist circumference, triglycerides, and $\gamma$-glutamyl transpeptidase. A study on 8626 patients determined the cutoff value of FLI in the evaluation of middle-aged and elderly patients with NAFLD. ${ }^{24}$ A cutoff value of 30 was found to be promising in the identification of patients with NAFLD with $80 \%$ sensitivity, $72 \%$ specificity, giving an AUROC of $0.83 .{ }^{24} \mathrm{FLI}$ is a practicable computing tool because it uses clinical and laboratory values that are readily performed in both inpatient and outpatient settings which enhances its applicability. This allows for effective screening of patients at risk of developing the disease and subsequent introduction of lifestyle modifications that can curb the development and/or progression of this ailment. It can also help in siphoning candidates with suspected NAFLD who can then take part in research models that target further screening, investigations and treatment. ${ }^{25}$

\section{Fibro test}

FibroTest is a noninvasive panel of serum markers to predict liver fibrosis with high NPV in advanced liver fibrosis. ${ }^{26-29}$ The serum markers in this panel are haptoglobin, alpha2 microglobulin, total bilirubin, $\gamma$-glutamyl transpeptidase, and apolipoprotein A1. A recent study used FibroTest to predict advanced fibrosis in NAFLD. ${ }^{30}$ The authors found AURCO of 0.81-0.92 in detecting F3-4 fibrosis and $0.75-0.86$ in predicting F2-4. They determined the cutoff value of 0.30 and 0.70 for advanced liver fibrosis with $90 \%$ NPV and $73 \%$ PPV. The diagnostic performance of FibroTest was evaluated in a study of 600 biopsy-proven NAFLD patient by comparing FibroTest with BRAD score, FIB-4 index, and NFS. ${ }^{31}$ The non-binary AUROC for FibroTest (0.877) was found to be superior to BRAD score (0.836), FIB-4 index $(0.845)$ and comparable with NFS $(0.866)^{31}$. Although FibroTest can detect liver fibrosis effectively, however, the routine application of this test is difficult due to unavailability of some of serum markers in most laboratories assay.

\section{Enhanced liver fibrosis panel (ELFP)}

ELFP is commercially available markers of matrix turnover including PIIINP, hyaluronic acid (HA) and tissue inhibitor of matrix metalloproteinase 1 (TIMP-1). A recent study utilized ELFP in NASH patients showed AURCO of 0.90 and 0.82 in detecting stage F3-4 and F2-4 of advanced fibrosis respectively. ${ }^{32}$ The ELFP cutoff value -0.2070 was found to have $61 \%$ sensitivity and $80 \%$ specificity to rule out liver fibrosis in NASH patients. ELFP is better diagnostic panel than NFS for detection of moderate fibrosis (AUROC 0.90 vs 0.86 ) and severe fibrosis (AUROC 0.93 vs 0.89 ), combination of these tests performs even better than individual test for detection of moderate (AUROC 0.93) and severe fibrosis (AUROC 0.98). ${ }^{32}$

\section{BRAD core}

BRAD core is utilized to detect advanced liver fibrosis F3-4 in NAFLD. The BRAD score is based on BMI, AST/ALT ratio, and status of type II diabetes in suspected patients with NAFLD. A cutoff score value $<2$ is a reasonable predictor in exclusion of advances fibrosis (NPV 95-97\%), while a cutoff score $>2$ is associated with advanced liver fibrosis F3-4 with sensitivity and specificity of $88 \%$ and $89 \%$ respectively, and an AURCO of $0.865 .{ }^{33}$ The BRAD has limited diagnostic value for detection of early stages of fibrosis and can only be utilized to predict severe fibrosis.

\section{Conclusion}

Diagnostic evaluation of patients with NAFLD/NASH is challenging. The scoring system is a promising tool for identification of NAFLD and its progression to NASH. Further validation studies are required for clinical utilization of scoring system for assessment of various stages of disease progression.

\section{Acknowledgments}

None

\section{Funding}

None

\section{Conflicts of interest}

The authors have no conflicts of interest. 


\section{Author's contribution}

a. Muhammad Nadeem Yousaf: Manuscript writing

b. Fizah S. Chaudhary: Manuscript and data review

c. Wajahat Z. Mehal: Manuscript writing and overall supervision.

\section{References}

1. Chalasani N, Younossi Z, Lavine JE, et al. The diagnosis and management of nonalcoholic fatty liver disease: Practice guidance from the American Association for the Study of Liver Diseases. Hepatology.. 2018;67(1):328-357.

2. EASL-EASD-EASO. EASL-EASD-EASO Clinical Practice Guidelines for the management of non-alcoholic fatty liver disease. $J$ Hepatol.. 2016;64(6):1388-1402.

3. Loomba R, Sanyal AJ. The global NAFLD epidemic. Nat Rev Gastroenterol Hepatol.. 2013;10(11):686-690.

4. Spengler EK, Loomba R. Recommendations for Diagnosis, Referral for Liver Biopsy, and Treatment of NAFLD and NASH. Mayo Clin Proc.. 2015;90(9):1233-1246.

5. Vernon G, Baranova A, Younossi ZM. Systematic review: the epidemiology and natural history of non-alcoholic fatty liver disease and non-alcoholic steatohepatitis in adults. Aliment Pharmacol Ther.. 2011;34(3):274-285.

6. Fraser A, Longnecker MP, Lawlor DA. Prevalence of elevated alanine aminotransferase among US adolescents and associated factors: NHANES 1999-2004. Gastroenterology.. 2007;133(6):1814-1820.

7. Williams CD, Stengel J, Asike MI, et al. Prevalence of nonalcoholic fatty liver disease and nonalcoholic steatohepatitis among a largely middleaged population utilizing ultrasound and liver biopsy: a prospective study. Gastroenterology.. 2011;140(1):124-131.

8. Wong VW, Wong GL, Choi PC, et al. Disease progression of nonalcoholic fatty liver disease: a prospective study with paired liver biopsies at 3 years. Gut. 2010;59:969-974.

9. Browning JD, Szczepaniak LS, Dobbins R, et al. Prevalence of hepatic steatosis in an urban population in the United States: impact of ethnicity. Hepatology. 2004;40:1387-1395.

10. Machado MV, Cortez-Pinto H. Non-alcoholic fatty liver disease: what the clinician needs to know. World J Gastroenterol. 2014;20(36):1295612980.

11. Charlton MR, Burns JM, Pedersen RA, et al. Frequency and outcomes of liver transplantation for nonalcoholic steatohepatitis in the United States. Gastroenterology. 2011;141:1249-1253.

12. Bensaid S, Kachenoura A, Costet N, et al. Early diagnosis of NAFLDNASH transition using mid infrared spectroscopy. Conf Proc IEEE Eng Med Biol Soc. 2016;2016:3602-3605.

13. Musso G, Gambino R, Cassader M, et al. Meta-analysis: natural history of non-alcoholic fatty liver disease (NAFLD) and diagnostic accuracy of non-invasive tests for liver disease severity. Ann Med. 2011;43(8):617649.

14. Soderberg C, Stal P, Askling J, et al. Decreased survival of subjects with elevated liver function tests during a. 28-year follow-up. Hepatology. 2010;51(2):595-602.

15. Ong JP, Pitts A, Younossi ZM. Increased overall mortality and liverrelated mortality in non-alcoholic fatty liver disease. $J$ Hepatol. 2008;49(4):608-612.

16. Dunn W, Xu R, Wingard DL, et al. Suspected nonalcoholic fatty liver disease and mortality risk in a population-based cohort study. Am J Gastroenterol. 2008;103(9):2263-2271.
17. Rafiq N, Bai C, Fang Y, et al. Long-term follow-up of patients with nonalcoholic fatty liver. Clin Gastroenterol Hepatol. 2009;7(2):234 238

18. Sorensen HT, Mellemkjaer L, Jepsen P, et al. Risk of cancer in patients hospitalized with fatty liver: a Danish cohort study. J Clin Gastroenterol. 2003;36(4):356-359.

19. Angulo P, Hui JM, Marchesini G, et al. The NAFLD fibrosis score: a noninvasive system that identifies liver fibrosis in patients with NAFLD. Hepatology. 2007;45(4):846-854.

20. Kobayashi N, Kumada T, Toyoda H, et al. Ability of Cytokeratin-18 Fragments and FIB-4 Index to Diagnose Overall and Mild Fibrosis Nonalcoholic Steatohepatitis in Japanese Nonalcoholic Fatty Liver Disease Patients. Dig Dis. 2017;35(6):521-530.

21. Kaswala DH, Lai M, Afdhal NH. Fibrosis Assessment in Nonalcoholic Fatty Liver Disease (NAFLD) in. 2016. Dig Dis Sci. 2016;61(5):13561364.

22. Imajo K, Kessoku T, Honda Y, et al. Magnetic Resonance Imaging More Accurately Classifies Steatosis and Fibrosis in Patients With Nonalcoholic Fatty Liver Disease Than Transient Elastography. Gastroenterology. 2016;150(3):626-637.e7.

23. Yang $\mathrm{H}$, Chen $\mathrm{G}$, Song $\mathrm{C}$, et al. A novel index including SNPs for the screening of nonalcoholic fatty liver disease among elder Chinese: A population-based study. Medicine. 2018;97(13):e0272-e0272.

24. Huang X, Xu M, Chen Y, et al. Validation of the Fatty Liver Index for Nonalcoholic Fatty Liver Disease in Middle-Aged and Elderly Chinese. Medicine. 2015;94(40):e1682-e1682.

25. Bedogni G, Bellentani S, Miglioli L, et al. The Fatty Liver Index: a simple and accurate predictor of hepatic steatosis in the general population. BMC Gastroenterol. 2006;6:33.

26. Pradat P, Alberti A, Poynard T, et al. Predictive value of ALT levels for histologic findings in chronic hepatitis $\mathrm{C}$ : a European collaborative study. Hepatology. 2002;36(4):973-977.

27. Myers RP, Benhamou Y, Imbert-Bismut F, et al. Serum biochemical markers accurately predict liver fibrosis in HIV and hepatitis $\mathrm{C}$ virus co-infected patients. Aids. 2003;17(5):721-725.

28. Myers RP, Tainturier MH, Ratziu V, et al. Prediction of liver histological lesions with biochemical markers in patients with chronic hepatitis B. $J$ Hepatol. 2003;39(2):222-230.

29. Naveau S, Raynard B, Ratziu V, et al. Biomarkers for the prediction of liver fibrosis in patients with chronic alcoholic liver disease. Clin Gastroenterol Hepatol. 2005;3(2):167-174.

30. Ratziu V, Massard J, Charlotte F, et al. Diagnostic value of biochemical markers (FibroTest-FibroSURE) for the prediction of liver fibrosis in patients with non-alcoholic fatty liver disease. BMC Gastroenterol. 2006;6:6.

31. Munteanu M, Tiniakos D, Anstee Q, et al. Diagnostic performance of FibroTest, SteatoTest and ActiTest in patients with NAFLD using the SAF score as histological reference. Aliment Pharmacol Ther. 2016;44(8):877-889.

32. Guha IN, Parkes J, Roderick P, et al. Noninvasive markers of fibrosis in nonalcoholic fatty liver disease: Validating the European Liver Fibrosis Panel and exploring simple markers. Hepatology. 2008;47(2):455-460.

33. Cichoz-Lach H, Celinski K, Prozorow-Krol B, et al. The BARD score and the NAFLD fibrosis score in the assessment of advanced liver fibrosis in nonalcoholic fatty liver disease. Med Sci Monit. 2012;18(12):CR735CR740. 